\title{
CULTURA DE EMPRESA Y MULTINACIONALES: EL CASO DE MAERSK ESPAÑA
}

\section{ENTERPRISE CULTURE AND MULTINATIONAL COMPANIES: THE CASE OF MAERSK SPAIN}

\section{RESUMEN}

Dentro del ámbito de la Sociología de las Organizaciones, la cultura empresarial se ha abierto un interesante campo desde la década de los 80, a partir de la cual se empezó a confiar en la posibilidad de dirigir grandes compañías con el respaldo de un conocimiento más íntimo de su cultura. Por lo tanto, el conjunto de disciplinas que aborda la teoría organizacional se ve atraído por su estudio, dada la demostrada utilidad de estandarizar comportamientos dentro de la compañía, así como la posible alteración de la propia cultura para repercutir positivamente en producción y beneficios.

Estas premisas nos llevaron al estudio de caso de Maersk España en Algeciras. Para tal efecto, abordamos la realidad laboral de la compañía, generando un modelo propio de análisis que nos ha facilitado retratar su cultura empresarial, aplicando distintas técnicas cuantitativas y cualitativas. Este diagnóstico nos ha permitido conocer las consecuencias de la integración de distintas subculturas del trabajo -habitual en multinacionales-, al entrar en el estudio de contrastes esenciales, como el que se produce entre la cultura empresarial danesa (procedencia) y andaluza (destino), así como las peculiaridades de su adaptación al entorno local (Campo de Gibraltar).

\section{PALABRAS CLAVE}

Organizaciones, cultura empresarial, sociología del trabajo, multinacionales.

\begin{abstract}
Within the scope of the Sociology of the Organizations, the enterprise culture has opened an interesting field from the decade of the 80 . Discipline began to trust the possibility of directing great companies with the support of a more intimate knowledge of his culture. Therefore, the set of disciplines that approach the organisational theory was attracted by their study, given the demonstrated utility to standardize behaviours within the company, as well as the possible alteration of the own culture to repel in production and benefits. These premises took us to the study of case of Maersk Spain in Algeciras. We approached the reality of the company, generating an own model of analysis that it has facilitated to us to explore its enterprise culture, applying different quantitative and qualitative techniques. This diagnosis has allowed us to know the consequences of the integration of different work subcultures -
\end{abstract}


habitual in multinationals-, when entering the study of essential distinctions, like which it takes place between the Danish enterprise culture (origin) and Andalusian (destiny), as well as the peculiarities from its adaptation the local surroundings (Campo de Gibraltar).

\section{KEYWORDS}

Organisations, enterprise culture, sociology of work, multinational companies.

\section{PLANTEAMIENTO DE LA INVESTIGACIÓN Y ANTECEDENTES TEÓRICOS}

Desde que surgió el interés por el estudio de la cultura de empresa en la década de los ochenta tras las aportaciones de pioneros como Deal y Kennedy (1985) u Ouchi (1984a), este campo ha experimentado un notable desarrollo en la ciencia social. La tendencia ha respondido a una demanda creciente en el sistema mundial de respuestas a nuevas incógnitas. ¿Qué hacen las organizaciones excelentes que no hagan las demás? ¿Hasta que punto un compromiso extra en los trabajadores puede generar valor añadido? ¿Cómo generar ese compromiso?

Sin embargo, el reciente desarrollo de la rama no ha desembocado en demasiados estudios de caso sobre "organizaciones excelentes".

Evidentemente, la causa está en las dificultades para penetrar en un mundo opaco, cargado de secretismo y presionado por las amenazas presentes en el entorno. Esta circunstancia acrecentó mi interés por indagar en una de las mayores multinacionales del mundo: el grupo A.P. Möller Maersk. Una de sus principales terminales a nivel mundial se encuentra en Algeciras y podía ser de utilidad para ampliar el abanico de estudios de caso sobre organizaciones en el ámbito de la cultura. El objetivo de mi trabajo es exponerles los resultados obtenidos en el mencionado estudio de caso -un análisis de la cultura de empresa-, que pretende realizar nuevas aportaciones al campo de la sociología de la organización. La investigación ha sido realizada en el marco de un convenio de colaboración científica que la Universidad de Granada firmó con Maersk España, S.A. en el año 2000.

La compañía danesa, fundada en Svenborg en 1904, es actualmente líder mundial en el sector del transporte, siendo su gran aportación a la economía internacional el desarrollo del transporte por tierra y mar de contenedores. La compañía se caracteriza por generar una imagen pública de extrema discreción, pasando desapercibida por el ciudadano de a pie. Dada su política corporativa, de escasa proyección al público, la compañía no ha generado apenas literatura sobre sí misma, con la salvedad de la historia de la compañía que escribiera Ove Homby en 1986 y que ha sido utilizada como un documento de difusión interna. Esta circunstancia animó a ambas partes (empresa e investigador) a generar alguna literatura alternativa que facilitará, en este caso, el conocimiento de la visión externa e interna de la compañía.

Nuestro acercamiento a la multinacional Maersk es, en parte, consecuencia de la euforia científica desatada hacia la cultura organizacional en los últimos tiempos. Estamos ante una vertiente necesitada de nuevos estudios de caso que permitan a la ciencia social obtener experiencias que impulsen las construcciones de nuevas teorías. En el caso español, se han realizado algunas aportaciones muy meritorias en este sentido, con trabajos 
como el realizado por Garvía sobre la Once en 1997 o el de Sala Schnorkowski sobre el cambio cultural en Renfe en 2000. Por su parte, la gran terminal de Maersk ubicada en la puerta del mediterráneo (Algeciras) se presentaba como un buen laboratorio para penetrar en cuestiones como la aculturación, difusión cultural, subculturas profesionales y de clase, así como conflicto organizacional.

El interés científico despertado en torno a la cultura organizacional responde a las difíciles circunstancias industriales y económicas experimentadas por Europa y Estados Unidos en las décadas de los 70 y 80 . Asimismo, la posibilidad de controlar la conducta grupal de las personas a través de la cultura era un tema que estaba ya presente en otros ámbitos de las ciencias sociales. Geertz se refiere así al fenómeno en 1973 en su trabajo La interpretación de las culturas: "cuando se la concibe (a la cultura) como una serie de dispositivos simbólicos para controlar la conducta, como una serie de fuentes extrasomáticas de información, la cultura suministra el vínculo entre lo que los hombres son intrínsecamente capaces de llegar a ser y lo que realmente llegan a ser uno por uno. Llegar a ser humano es llegar a ser individuo y llegamos a ser individuos guiados por esquemas culturales, por sistemas de significación históricamente creados en virtud de los cuales formamos, ordenamos, sustentamos y dirigimos nuestras vidas (...) ${ }^{\mathrm{ii}}$,

Desde la perspectiva de Coller y Garvía (2004: 59-60), el enfoque cultural surge en el ámbito organizacional ante la aparente escasez de resultados de las investigaciones comparativas y de base cuantitativa de las décadas de los sesenta y setenta del siglo XX, orientadas a explicar la estructura de la organización a través de variables como el tamaño, la tecnología y el entorno. En este ambiente de expectativas defraudadas, los departamentos de sociología dejarán el campo de estudio a dos comunidades científicas con tradiciones, metodología y herramientas conceptuales bien distintas: la de los sociólogos de orientación funcionalista, preocupados por explicar la eficiencia organizacional y que trasladaron su doctrina a las escuelas de negocios y, frente a ella, la de los antropólogos y psicólogos sociales orientados al estudio de la dimensión cognitiva y simbólica.

Por su parte, Gómez Cabranes resumió así, en 1994, los tres factores clave que desencadenan el creciente interés por la cultura organizacional desde los 80. En primer lugar, El éxito de la empresa japonesa en su expansión internacional que despertará un interés científico generalizado. En segundo lugar, la creciente incertidumbre del entorno competitivo de la actividad empresarial impulsa la reflexión sobre la identidad corporativa. Por último, destaca la aparición de organizaciones que alcanzan la excelencia fundamentándose en una cultura fuerte: Hewlett-Packard, The Body Shop o Mondragón. Los principios de dirección que han llevado al éxito a estas tres compañías desbordaron las expectativas de la competencia. No obstante, surge la tendencia generalizada a la imitación de culturas excelentes: por algo los demás lo hicieron mejor que nosotros, aprendamos de ellos.

El concepto cultura organizacional ha entrado con fuerza en el mundo de la administración de empresas. Las nuevas tendencias empresariales no encontraban explicaciones suficientes en la teoría de la administración existente y comienzan a buscarse nuevas referencias desde los 60. En este contexto emergen los estudios sobre hospitales psiquiátricos de Garfinkel (1968) y de Goffman (1970), tratando de comprender las circunstancias que generaban cuadros problemáticos en sus organizaciones. Por otra parte, autores como Merton (1980a) o Perrow (1984), centraron su atención en cuestiones tan determinantes como el conflicto y el poder en las organizaciones. El conocimiento, control 
y cambio de la cultura de la organización empiezan a concebirse como armas competitivas de la nueva empresa innovadora, tal y como destacan los primeros trabajos de Peters y Waterman (1984) Smircich (1994) o Scheim (1995). En este sentido, penetrarán, cada vez con más fuerza, los estudios que resaltan el dominio de la organización japonesa y la exportación de su cultura al mundo, con trabajos como los de Ouchi (1984), Reitzperger (1991a) o Wei Choo (1999). Estos trabajos darán paso a un creciente interés por la interculturalidad e internacionalidad en las organizaciones, destacando en este ámbito los trabajos de Hofstede (1999a; 2002).

Las promesas que rodearon al factor cultural en el ámbito empresarial no se han cumplido totalmente, si bien en las dos últimas décadas ha crecido en protagonismo en el ámbito de los estudios organizacionales (Lucas Marín: 2002: 192). Asimismo la presencia académica y científica de la rama en Estados Unidos, Japón y Europa ha crecido notoriamente. Por lo tanto, el presente análisis de la cultura organizacional de Maersk se sitúa en la onda expansiva de esta tendencia.

Al tratarse de la filial de una multinacional danesa situada en el sur de España, el contraste existente con la cultura del entorno en el que opera y su estilo de gestión, contribuye a resaltar más las particularidades que han convertido a esta compañía, miembro del Grupo A.P. Möller, en una de las más importantes multinacionales del mundo. Maersk está considerada actualmente en todo un referente en excelencia en el sector portuario y del transporte, circunstancias que han fomentado el interés en su análisis.

En definitiva, la elasticidad de las organizaciones, demostrada por las experiencias precedentes, permite copiar los modelos que hicieron triunfar a los mitos del management, con mayor o menor éxito. Podemos aprender de "los más grandes" de la historia de las organizaciones, buscando determinados fragmentos culturales exportables, incluso comportamientos ajenos a la cultura local pueden ser impuestos en la organización a través de una cultura fuerte (véase el caso de la industria estadounidense del automóvil en la actualidad, incorporando técnicas toyotistas a su programación) ${ }^{\mathrm{iii}}$. En la sociología actual se resalta, cada vez más, la posibilidad de hacer renacer la vieja cultura de clan sobre las despersonalizadas organizaciones occidentales modernas, gestadas en principios individualistas en los últimos 30 años, confirmándose así que el entorno de la teoría organizacional se convierte en un mundo lleno de paradojas y de caminos de ida y vuelta.

\section{OBJETIVOS Y METODOLOGÍA}

Partiendo de las mencionadas consideraciones teóricas, en este trabajo nos hemos propuesto analizar la cultura organizacional de Maersk España en su peculiar adaptación a la comarca del Campo de Gibraltar tras el desarrollo de un completo trabajo de campo que nos permitirá obtener interesantes conclusiones sobre cuestiones como el impacto de las culturas nacionales sobre el comportamiento de sus filiales (difusión cultural), el fenómeno de aculturación producido por el contacto con la cultura local y la fusión cultural tras la operación en la que Maersk absorbe a Sealand en 1999, compañía norteamericana que aplicaba diferentes métodos y técnicas al trabajo. Por otra parte, debemos destacar las interesantes perspectivas científicas de cara a la obtención de conclusiones sobre las diferencias de conducta laboral entre los distintos grupos de trabajadores implicados en el funcionamiento de la terminal. 
Para la elaboración de un modelo que me permitiera profundizar en el análisis de la multinacional Maersk, he considerado las tres grandes perspectivas con el objeto de integrar en él sus puntos de vista: enfoque del entorno sociocultural, el liderazgo y la perspectiva de los empleados ${ }^{\text {iv }}$. Los factores que determinan este interés por los tres enfoques son, en primer lugar, la experiencia contemplada en estudios de caso realizados sobre cultura organizacional en multinacionales, que han demostrado la aplicabilidad y utilidad de todos ellos. Y, en segundo lugar, la complejidad de la situación vivida por la empresa en el Campo de Gibraltar muestra tal amplitud de facetas de la cultura organizacional que invita a dar respuestas desde los tres enfoques predominantes ya mencionados. La triangulación de estas perspectivas de los enfoques me ha permitido abrir más aún los ángulos de visión de la investigación.

El objetivo general de esta investigación es conocer los comportamientos culturales propios de la compañía, indagando en su origen, causas y utilidades. De esta forma, analizaremos las diferencias de comportamiento asociadas a las subculturas existentes entre los diferentes sectores y clases que componen la compañía. Por otra parte, se identificaron otros cuatro objetivos operativos para emprender la investigación:

- Crear un modelo de operacionalización de variables propio para el análisis organizacional de la compañía, adaptado a sus propias características (fase exploratoria).

- Realizar una auditoria de la cultura organizacional de la compañía, siguiendo el modelo anteriormente indicado, combinando técnicas cuantitativas y cualitativas para la producción de datos propios sobre la terminal de Algeciras.

- Obtener, a través del posterior análisis de los datos, algunas conclusiones sobre sus peculiaridades culturales, así como presentar a la compañía una herramienta técnicamente fundamentada para la autocrítica.

- Realizar aportaciones a la disciplina de la sociología de las organizaciones, basadas en aquellos aspectos recogidos en el caso que puedan ser útiles, corroborando los resultados obtenidos en otras experiencias investigadoras o aportando nuevas visiones y las consiguientes hipótesis.

La metodología establecida está inspirada en modelos ya utilizados por otros sociólogos que han aplicado cuadros de operacionalización de variables ${ }^{\mathrm{v}}$ para retratar la cultura organizacional. Dada la riqueza y peculiaridad del caso Maersk, he optado por aprender e interpretar los modelos anteriores para la creación de un modelo propio de análisis de aplicación exclusiva a este estudio de caso. Se trata de una investigación extensa que ha permitido la elaboración de un modelo adaptado a la peculiaridad del estudio "en proceso", es decir, tras obtener los primeros resultados de la fase exploratoria. El esquema se articula en dimensiones, subdimensiones e indicadores que tratan de describir el conjunto de la organización y que pueden ser medias aplicando técnicas cuantitativas (encuesta y análisis de fuentes estadísticas) y cualitativas (entrevistas en profundidad, grupos de discusión, observación directa y análisis documental). De esta manera podremos calibrar cuestiones como la existencia de una cultura fuerte o débil, el estilo de comunicación y cultura formativa, entre otros aspectos de interés. Para facilitar el 
análisis, debemos diferenciar entre dos grupos sociales de diferente naturaleza encuadrados en la misma organización:

"Cuello blanco"vi: conglomerado compuesto por los directivos, mandos intermedios y administrativos de la terminal. Albergan en su currículum conocimiento de idiomas, informática y/o formación universitaria.

"Cuello azul": conjunto formado por los operarios de cualificación media o baja que trabajan en la terminal. Aportan formación técnica para realización de trabajos de mantenimiento, destrezas en el manejo de máquinas y conducción de vehículos de transporte.

Las diferencias entre ambos estratos no se basan tanto en el salario como en otros aspectos. Se trata de diferentes estructuras laborales, estilos de vida, motivaciones y caracteres formativos. Ambos conglomerados manifiestan intereses, cuando menos diferenciados, y en algunos frentes también contrapuestos. Por lo tanto, se inicia una primera aproximación para obtención de información primaria utilizando dos grupos de discusión en la fase exploratoria (ver cuadro 1).

Cuadro 1. Planificación de la investigación

\begin{tabular}{|c|c|}
\hline PRIMERA FASE (EXPLORATORIA) & \\
\hline TÉCNICA & APLICACIÓN \\
\hline GRUPO DISCUSIÓN “BLANCOS” & Mandos intermedios y técnicos \\
\hline GRUPO DISCUSIÓN “AZULES” & $\begin{array}{l}\text { Operarios y trabajadores de baja } \\
\text { o media cualificación }\end{array}$ \\
\hline
\end{tabular}

Tras el análisis de los resultados de la primera fase se ha diseñado el cuadro guía de operacionalización de variables, el diseño de la investigación se ha retroalimentado, permitiendo crear un modelo propio de análisis cultural, adaptado íntegramente a nuestras necesidades investigadoras.

\begin{tabular}{|l|l|}
\hline \multicolumn{1}{|l|}{ SEGUNDA FASE (DESCRIPTIVA) } \\
\hline TÉCNICA
\end{tabular}

El nuevo modelo se ha usado como patrón esencial para estructurar la aplicación de las técnicas de investigación en el resto del trabajo de campo (fase descriptiva). En esta 
segunda fase se planificó la inclusión de tres grupos de discusión que, en este caso, responden a otros criterios de división, con objeto de ampliar la perspectiva de la investigación. En esta fase cumplimentaremos los anteriores puntos de vista, contando con tres divisiones en base a la estructura departamental de la empresa: departamento de mantenimiento, departamento de marina y departamentos de administración y terminal.

\section{ANÁLISIS DE LOS RESULTADOS}

Mi exposición de los resultados más significativos del estudio de caso incidirá en los siguientes aspectos: por una parte, la clasificación de la cultura analizada en base a las principales referencias teóricas, sus rasgos y peculiaridades (¿en qué es distinta a las demás?) y, en un segundo lugar, la diferenciación de los aspectos mejorables en la organización de sus puntos fuertes ${ }^{\text {vii }}$.

\subsection{La cultura Maersk: clasificación, rasgos y peculiaridades}

La sociología de las organizaciones ha establecido distintas tipologías para facilitar la labor de los analistas a la hora de clasificar las culturas: distinción en el eje fuerte/débil (Andrews y Basler, 1993), según su orientación en los ejes tiempo y riesgo (Deal y Kennedy, 1985) y las tipologías mitológicas de Handy (1976).

Resaltaré, en primer lugar, distinciones en la caracterización "fuerte/ débil""viii, para obtener referentes que permitan acercarnos a la esencia cultural de la organización, así como las implicaciones que este rasgo tiene en la estratificación social y en la distribución del poder. Al clasificar la "cultura Maersk" en el mencionado eje "fuerte/ débil" nos encontramos ante circunstancias dispares en función de los indicadores analizados. Se establecieron cuatro ejes en los que se confrontaban aspectos propios de una cultura fuerte frente a rasgos de culturas débiles. En un intento de medición objetivo, recurrimos a la encuesta realizada en la terminal, en la que se utilizaron escalas de Likert con puntuaciones de "1 a 5" para medir la flexibilidad, la identificación con la multinacional, la autonomía, y la participación. En este caso la correspondencia de ambos extremos a la dureza o debilidad de la cultura depende de la afirmación utilizada para realizar la medición. A continuación, comparamos en el cuadro 2 la media obtenida sobre las 97 respuestas recibidas, obteniendo los siguientes resultados:

Cuadro 2. Frases guía e indicadores relativos a la fortaleza de la cultura

\begin{tabular}{|l|}
\hline P.3 En lo personal me siento identificado con la multinacional MAERSK. \\
\hline P.6 Los trabajadores participamos en las decisiones que afectan a las labores cotidianas. \\
\hline P.8 Se nos considera autónomos, responsables y, como consecuencia, independientes. \\
\hline P.17 Creo que nuestros empleos son, en líneas generales, seguros y estables. \\
\hline P.23 En MAERSK somos flexibles, versátiles y con capacidad de adaptación a los cambios. \\
\hline
\end{tabular}

Medias obtenidas (Entre 1 "en desacuerdo" y 5 "totalmente de acuerdo")

Con la medición obtenida a través de estos resultados obtenemos algunas referencias claras del grado de dureza de la cultura Maersk. La compañía aparece en una posición intermedia con una leve tendencia a la dureza, ya que se observa que en los rasgos en los que se presenta como "fuerte" (estabilidad laboral e identificación) las puntuaciones 
son más contundentes que los rasgos que indican debilidad (flexibilidad y participación).

\begin{tabular}{|l|c|c|}
\hline \multicolumn{1}{|c|}{ VALOR } & MEDIA & DIAGNÓSTICO \\
\hline P.3 Identificación & 3,55 & (FUERTE) \\
P.6 Participación & 2,50 & (DÉBIL) \\
P.8 Autonomía & 3,00 & (NEUTRO) \\
P.17 Estabilidad laboral & 4,16 & (FUERTE) \\
P.23 Flexibilidad & 3,05 & (DÉBIL) \\
\hline
\end{tabular}

Fuente: Vallejo Peña, A. El análisis de la cultura de una multinacional. El caso Maersk. Granada. Comares. 2007

A pesar del importante éxito obtenido por culturas fuertes en la economía mundial en las últimas décadas, no debemos incurrir en la falacia de pensar que estos sistemas culturales hacen a las organizaciones, necesariamente, más productivas y eficaces. Ante esta disyuntiva Andrews, S. y Basler, C. afirmaban en 1993: "las culturas que están más débilmente implantadas suelen ser también más flexibles porque los individuos incorporan elementos culturales de otros sitios. Las culturas débiles pueden garantizar una mayor independencia de criterio, aunque pueden ser también más conflictivas al generar mayor ambigüedad. Una cultura que no sirva de base para respuestas claras y compartidas a los desafíos del entorno (o sea, débil) aumenta la probabilidad de que los individuos recurran a sus propias experiencias, atribución de significados y mitos (independientemente de la organización) para elaborar sus respuestas." En determinados sectores, actuar con la flexibilidad que permite una cultura débil se traduce en comportamientos eficientes. Organizaciones como un centro universitario o una agencia de publicidad difícilmente sobrevivirían en sus respectivas actividades sin responder a este modelo.

Asimismo, entrando en las principales tipologías culturales aportadas por la literatura, la "cultura Maersk" presenta también rasgos de cultura de "juego duro", en la que no se asumen enormes riesgos en las decisiones cotidianas, pero las acciones son evaluadas rápidamente a través de un continuo feedback, que mantiene en tensión a la plantilla (Deal y Kennedy: 1985). Se incluyen en su perfil rasgos de cultura "orientada a los resultados", en la que se valora la capacidad de resolución de problemas y las normas quedan en un segundo plano a favor de la flexibilidad (Handy: 1976).

Entre los objetivos marcados en la investigación se incluye destacar las peculiaridades de la organización: ¿Por qué se caracteriza? ¿Qué la hace diferente? En un primer término, nuestro equipo midió el grado de identificación de los miembros de la terminal con la multinacional Maersk y se aprecia, claramente, en el trabajo de campo, un profundo sentimiento de identificación. El 88.7\% de los entrevistados se manifiesta entre los valores 3 y 5 de la escala ("de acuerdo" y "totalmente de acuerdo") y un 28,7 \% muestra el máximo grado de acuerdo y tan solo $4.3 \%$ está en desacuerdo con tal afirmación. Los datos demuestran como el sentimiento de identificación es similar entre los trabajadores de cuello blanco y azul, si bien se intensifica entre los que tienen su origen laboral en Maersk. Si bien es importante resaltar que la identificación se da fundamentalmente con el grupo de trabajo de Algeciras, mientras que la identificación con la multinacional de Copenhague es más tenue. Por otra parte, las referencias a la contribución de la compañía al desarrollo de la comarca y la participación de los miembros en el proyecto tienen una notoria presencia en el discurso. 
Al indagar en las circunstancias que pueden dañar la calidad de vida de los trabajadores resaltan las circunstancias de los directivos, mandos intermedios, técnicos y mecánicos soportan un alto estrés y afrontan fuertes responsabilidades. La dinámica de la actividad les obliga a mantenerse localizables 24 horas diarias para consultas y posibles emergencias. Esta circunstancia resta calidad de vida en los trabajadores, que en muchos casos viven materialmente "pegados" al teléfono. Se les exige atención inmediata y respuestas rápidas desde arriba. Se da una tendencia clara a personalizar los problemas, marcando claramente al trabajador su ámbito de responsabilidad. Las iniciales del empleado en el resguardo de un correo electrónico delatando un error cometido pueden ser utilizadas como arma arrojadiza. Si alguien necesita un dato desde Copenhague o Madrid hay que proporcionarlo, y si se exige una modificación habrá que ejecutarla. La encuesta realizada sitúa al 70,9\% de las respuestas entre las opciones "de acuerdo"/ "totalmente de acuerdo" ante la afirmación "la empresa fomenta la autonomía y la responsabilidad de los trabajadores".

Por otra parte, las exigencias del sector del transporte marítimo implican que los técnicos se vean continuamente desbordados por obligadas improvisaciones:

"Ellos son los que saben de estos temas y esto es un puerto de destino ellos saben que el barco que llega no tiene este contenedor y lo cambian todo. Se altera el orden de trabajo y ellos tiene que estudiar el riesgo." GD3-30

El discurso generalizado muestra que la plantilla tiene asumida la separación entre concepción y ejecución, un claro rasgo taylorista. Los trabajos vienen suficientemente planificados y depurados desde arriba como para considerar las opiniones del operario.

Maersk es una compañía de talante previsor y planificador, si bien la obligación de ser competitivos en el sector y las vicisitudes del transporte marítimo hacen ineludible la reestructuración de cualquier planificación prevista:

"A veces tienes que tomar decisiones arriesgadas en poco tiempo y con riesgo y hay errores, claro que los hay. Ellos han estudiado el riesgo y después toman la decisión, pues ellos sabrán." GD3-31

En la misma línea de éste discurso, en el grupo de discusión del Departamento de Marina opinan así:

"Es un sistema comparable con cualquier cadena de producción pero con una gran diferencia, en otras cadenas está todo planificado y calculado y aquí no. Está casi todo sin previsión por lo intrínseco de este trabajo. Tú tienes dos grúas trabajando y una de ellas cargará un contenedor a las 9 de la mañana, la otra cargará un rato después. Bien, todo perfecto, lo que pasa es que una grúa se avería, ahora la planificación hay que cambiarla. Ese problema lo puedes tener en cada momento no te puedes poner a dar explicaciones a todo el mundo. Es difícil entender para el operario que ahora haya que coger el contenedor de abajo en lugar del de arriba, pero es así, porque ya no da tiempo a cambiar la planificación anterior" GD3-33

Estas circunstancias explican su flexibilidad en el trabajo, fruto de la presión del entorno. Existe la impresión generalizada de que los componentes de la empresa, socializados en una cultura muy dinámica, son fácilmente flexibles y adaptables a las circunstancias. En la multinacional nórdica los comunicados se distribuyen con gran 
rapidez y los cambios improvisados sobre la planificación prevista son frecuentes. En el transporte marítimo impera la atención rápida al cliente, que se juega dinero y compromisos en los transbordos. Al mismo tiempo el personal con responsabilidad es consciente de que, en ocasiones, no tiene demasiado tiempo para meditar sus decisiones y, por lo tanto, se le exige una gran capacidad de improvisación. En Maersk las planificaciones previstas están sometidas a repentinas alteraciones por las circunstancias variables propias de la actividad. El 61,7 \% de los encuestados está "bastante" o "totalmente de acuerdo" con la afirmación: "En Maersk somos versátiles, flexibles y con capacidad de adaptación a los cambios" mientras que sólo un 3,2 \% se muestra "en desacuerdo". El consenso existente en la terminal respecto a la flexibilidad de la organización es contundente.

Por otra parte, la estabilidad de los contratos de la terminal ha contribuido a fortalecer la "cultura Maersk", de igual forma que el planteamiento de la multinacional a nivel mundial de fomentar una carrera vitalicia a través de altas inversiones en formación. Los estudios realizados en psicología del trabajo demuestran los beneficios que se obtienen para el trabajador y la compañía a través de la seguridad laboral: concentración, regularidad e integración en el equipo, entre otros. Sin embargo, debemos considerar también las disfunciones que conlleva la seguridad en el puesto. El trabajo de campo ha delatado algunos síntomas de relajación y los testimonios de los trabajadores indican que se puede generar un "efecto apoltronamiento" en determinados puestos. Los casos que se presentan son cubiertos por la cadena de trabajo, suficientemente consistente para cubrir algunas deficiencias.

La conflictividad laboral en Maersk presenta un panorama bastante árido, en la línea de la marcada tradición de "sindicalismo tenaz" en el sector portuario. La dirección vive en continua tensión con los sindicatos y los representantes de los trabajadores, de forma que en la terminal acostumbran a soportar varias huelgas al año. Los directivos de la empresa insisten en que casi lo único que pueden ofrecer para contrarrestar las continuas reivindicaciones es dinero o tiempo (vacaciones y horas de servicio). Se entra en un juego que ellos califican como "negociación sin contraprestaciones" que ha sorprendido a algunos de los integrantes de la facción danesa, acostumbrados a un sindicalismo de menor agresividad. Un porcentaje significativo de los trabajadores considera que "la tensión y la conflictividad laboral en torno a Maersk son fuertes". El 34 \% manifestó estar "de acuerdo" y un 34,5 \% se mostraba bastante o "totalmente de acuerdo". La percepción de un panorama de tensión es generalizable tanto para el grupo de técnicos y directivos como para los operarios de la empresa.

Abordando la formación en la compañía como dimensión del estudio, la opinión generalizada en la terminal de Algeciras coincide en la idea de que en la compañía se invierte en formación y la concienciación sobre su utilidad es significativa. Sin embargo existe un problema de tiempo, un bien escaso en la empresa, que genera presión en los procesos de aprendizaje. La mayor parte del personal de la terminal tiene la sensación de que en Maersk la formación se adquiere en un "tren en marcha" y que las circunstancias laborales obligan en ocasiones a aprender a base de "encontronazos" con nuevos programas o materiales. Por otra parte, esta escasez de tiempo genera una percepción de pérdida y mal aprovechamiento de las horas dedicadas a formación -sensación naturalmente errónea- que fomenta un espíritu crítico hacía las acciones emprendidas. La encuesta realizada delata algunas lagunas en la formación recibida en algunos puestos de la compañía, encontrándose siempre el factor tiempo como la principal barrera. 


\subsection{Aspectos mejorables y fortalezas}

El management moderno se ha visto abordado por una nueva tendencia que cuenta con su corroboración científica en la teoría organizacional: la escucha activa (Kotler: 2001). La empresa moderna debe cultivar su oído e incorporar la escucha a sus hábitos y costumbres. A través de ella potenciaremos la obtención de información extra en forma de feedback. El enfoque institucional nos destacaba estos aspectos desde la difundida teoría de la racionalidad limitada (March y Simon: 1958), en la que se nos recordaba las imperfecciones de nuestras decisiones organizacionales, asumidas siempre sin la información deseada sobre el mercado y el entorno. No obstante, el "arte del management" estaría en ser hábiles tomadores de decisiones aún reconociendo la existencia de zonas de incertidumbre no controladas por el directivo.

Tratando de evitar planteamientos pesimistas, la moderna dirección de empresas puede recurrir a la explotación de "nuevas" fuentes de información despreciadas e ignoradas hasta los 80. Desde el marketing hasta la gestión de recursos humanos, una nueva tendencia inunda las lecciones impartidas en escuelas de negocios de todo el mundo: escuchen a su entorno, a los clientes externos y, también, a los internos. Cualquier transacción en una simple venta de un bien o servicio puede aprovecharse para obtener información valiosa para la organización. Todos somos conscientes de ser utilizados como consumidores o clientes para la obtención de datos personales que completan masivos trabajo de campo. Rellenamos cotidianamente formularios o pequeñas encuestas para contratar servicios con las que las empresas recogen datos de los que infieren nuestra clase social, gustos, preferencias y nivel de renta, entre otros. Algunas empresas adiestran a sus empleados para realizar periódicamente observaciones cualitativas sobre las reacciones de los consumidores tras la prestación de servicios (se trata de una técnica muy explotada en el sector turístico). Hasta la protesta de un cliente al girarse tras firmar la recepción de un pedido contiene información interesante para la organización. Los departamentos de reclamaciones, además de proporcionarnos datos sobre nuestras lagunas e imperfecciones, ofrecen una gran oportunidad para realizar escucha activa. Los sistemas para recoger y analizar estas informaciones no son demasiado costosos hoy día, y la empresa puede sacarle un gran rendimiento si la utiliza constructivamente (Kotler: 2001). De igual forma sucede con nuestros clientes internos, de los que obtenemos un feedback continuo. Las organizaciones han ido incorporando a su cultura la percepción de los departamentos a los que se prestan servicios como clientes de cuya satisfacción dependerá el funcionamiento armónico del sistema y por consiguiente, la producción.

El estilo directivo de Maersk se presenta como notoriamente conservador en estos aspectos. La empresa basa su confianza en los conocimientos científicos y técnicos, y no ha incorporado la "escucha activa" a todos los niveles en su cultura. Planteemos el caso del operario de una compañía se haya encargado del mantenimiento de una misma planta durante los últimos 25 años, y mientras ha coincidido con distintas generaciones de trabajadores empapándose de sus costumbres, hábitos, conversaciones y presenciando escenas en contextos que van de la desesperación a la euforia. En más de una ocasión habrá sido escogido por un directivo con ganas de desahogarse ante un ente anónimo, mostrando la presión soportada en el trabajo para explicarle su difícil situación, y éste le habrá escuchado estoicamente, de igual forma que nosotros lo habremos hecho con un frustrado compañero de viaje sentado en frente nuestra en un tren de pasajeros. El nivel 
formativo del operario no le permitiría en otras condiciones aportar demasiado, pero las circunstancias le harán distinguir y recordar la crisis económica del 73, la del 90 y la euforia económica de los últimos años de la década de los 90. Si planteamos a la dirección de una compañía -cualquiera que sea- la posibilidad de aprovechar testimonios vitales como el del mencionado operario, se podrá argumentar en su contra que se trata de un sencillo trabajador manual. En tal caso, quizás habría que plantear a la compañía que el conocimiento acuñado tras 25 años podría traducirse en consejos útiles para un joven directivo recién fichado.

La compañía danesa, al igual que tantas otras, puede obtener información extra de gran interés potenciando sus vías de comunicación internas y creando una cultura de escucha activa. Humberto Eco ya nos destacaba en 1977 la importancia de la "humildad científica" y la posibilidad de encontrar soluciones a través de la pluralidad de argumentos $^{\mathrm{x}}$. La atenta observación durante décadas se ha transforma en conocimientos que han llegado a asombrar a los científicos. Insistimos: el conocimiento profundo de las organizaciones no sólo es patrimonio de los accionistas, directivos o científicos. Debemos pensar entonces en aquellos empleados que llevan más de treinta años al servicio de una compañía.

Asimismo, este fomento de las actitudes comunicativas y constructivas podría facilitar el acercamiento entre la colonia danesa. Los resultados obtenidos confirman nuestras sospechas acerca de los efectos perversos del choque cultural. Se han generado algunas barreras entre ambos grupos que transmiten una sensación generalizada de distancia, quizás innecesaria. En los discursos profundos recogidos en el trabajo cualitativo, ambas partes reconocen algunas virtudes en el otro lado. Tal y como nos decía un directivo danés de la compañía:

"Desde luego que he visto virtudes en los españoles: con habilidades sociales, desenvueltos, con capacidad de improvisación ante los imprevistos... eso es muy importante en este trabajo. Se quedan fuera del horario sin ponerte las caras que te pondría un danés... Eso sí, tenemos la sensación de que tenemos que seguirles de cerca para que no nos mareen con sus improvisaciones e historias" (E8-112).

Un técnico cualificado nacional, por su parte, aportaba:

"Son serios y cumplidos (los daneses)... se lo piensan mucho antes de despedir a un trabajador, aunque haya hecho una barbaridad. Reconocen el trabajo y la capacidad. Esto es una sociedad de mérito, aunque a algunos no les gusta eso." (E4-52)

Entre los aspectos mejorables destaca, también, la posibilidad de fomentar un liderazgo más constructivo entre los directivos y mandos intermedios. Conducir un grupo y activar su motivación recurriendo a la asertividad y a refuerzos positivos puede mejorar el rendimiento colectivo. La compañía ha sobrevalorado aspectos como el tiempo y el dinero para mantener activos a sus trabajadores. Dichos factores son los más determinantes en cualquier actividad laboral, pero no los únicos que suman. De igual forma, la incorporación de nuevos empleados a una organización puede ser utilizada para considerar opiniones de alguien no "contaminado" por el ambiente de la empresa y que puede aportar argumentos menos subjetivos sobre las situaciones observadas La dirección de la compañía señalaba que estas circunstancias habían sido ya advertidas por una multinacional de consultoría que les diagnosticó recientemente (2004): se tiende a aplicar 
un estilo de liderazgo fragmentario y poco empático. Próximamente la compañía introducirá un programa formativo sobre liderazgo de grupos de trabajo. Esta política puede ser un buen comienzo.

"El liderazgo es como la belleza; difícil de definir; pero fácil de reconocer cuando uno lo ve. (...) Es posible que sea verdad que las empresas estén excesivamente dirigidas e insuficientemente lideradas."

(Bennis: 2000: 137)

"No estamos al nivel de motivación de años atrás, pero si se nota la experiencia".GD3-77

Por otra parte, los testimonios de la terminal han delatado la necesidad de reactivar la motivación existente en el equipo durante los primeros años de andadura en la compañía danesa en el Puerto Bahía de Algeciras. A finales de los 80 el equipo de trabajo era mucho más reducido y se generó un ambiente de cierta familiaridad, difícilmente reproducible en la terminal de la actualidad (286 personas, trabajando en la matriz). No obstante, aquel brillante clima social de fluido intercambio de favores y experiencias ha decaído, dando paso a relaciones interdepartamentales más frías y anónimas. Esta circunstancia es propia de compañías en plena expansión. De igual forma, el efecto novedoso de los trabajos ofrecidos entonces se ha diluido poco a poco y algunos puestos se ven marcados por la rutina, con el consiguiente coste motivacional para la empresa. Maersk deberá plantearse en los próximos años fomentar más aún la cohesión de los equipos de trabajo en la terminal. Si bien, dicha cohesión no es demasiado valorada por algunos técnicos y directivos. Al mismo tiempo, deberán plantearse acciones que mejoren la motivación del personal. Esto no implica, obviamente, que deba adaptar a su dirección todas las nuevas fórmulas de la gestión participativa, pero sí, al menos, las más adaptables a sus circunstancias

En cuanto a los aspectos favorables, debemos destacar que la terminal de Algeciras ha desarrollado notables habilidades en su gestión flexible. La empresa ha desarrollado una gran capacidad de improvisación en las decisiones operativas en respuesta al continuo estado de alarma que exige el sector. Las pruebas obtenidas delatan que Maersk es una empresa con excelentes reflejos. Este aspecto viene también potenciado por su tecnología, que facilita la implantación de una buena red de comunicación.

Al abordar el posible choque cultural generado por la convivencia entre los directivos y técnicos daneses (más poderosos) y la plantilla local (más numerosa), debemos destacar que el producto cultural de esta convivencia ha sido grato, al menos, desde el punto de vista económico. La empresa danesa está implantada en más de 100 países y los resultados de su filial algecireña, son óptimos en términos comparativos. Ciertamente, se han generado algunas asperezas por el contraste cultural tan notable existente entre la colonia danesa y la plaza andaluza, a lo que se suman las diferencias de estatus existentes entre ambos colectivos (favorable a los daneses). Si bien, en las entrevistas, ambas partes han reconocido, además de los defectos predominantes en el discurso, algunas significativas cualidades en la contraparte extranjera. Los españoles hemos destacado su capacidad de planificación y previsión, así como su reconocimiento a los méritos en el trabajo. Los daneses han señalado nuestra enorme capacidad de improvisación ante los imprevistos o situaciones límite.

Igualmente, se ha apreciado una importante integración de la cultura formativa en la compañía a todos los niveles. Si bien hemos topado continuamente con la dimensión 
formativa enfrentada a la dimensión temporal: ¿cómo sacar tiempo para formación? La mencionada formación ha sido identificada como una necesidad continua en la terminal, $y$ valorada por los diferentes colectivos que forman la plantilla, indiferentemente de su cualificación y estatus. Se reconocen, por otra parte, los esfuerzos inversores de la multinacional en formación, no tan productivos como se desearía por las dificultades organizativas que implica el trabajo a turnos y la escasez de tiempo. Asimismo, la plantilla reconoce la falta de implicación del equipo en estas actividades con un discurso marcadamente autocrítico. Solo desde la dirección se podrá crear una auténtica cultura de la formación, que implicaría al conjunto de los actores. Trabajar en este aspecto con los nuevos miembros que se incorporen a la plantilla será, naturalmente, determinante, ya que trasmitir una cultura formativa va mucho más allá de la organización de cursos. Las compañías que asientan en ella uno de sus pilares mentalizan a sus trabajadores desde su acceso a la empresa y crean cadenas activas de transmisión de conocimiento basadas en la actitud del equipo y con escasos costes económicos (Vallejo Peña: 2007). Una de las experiencias conocidas que ha consolidado la cadena de transmisión se da en el caso de la "cultura Cepsa", que la incluye entre sus axiomas, de forma que los nuevos candidatos se socializan escuchando repetitivamente slogans como: "la formación forma parte de mi puesto de trabajo" o "todos los días enseño algo, todos los días aprendo algo."xi

\section{CONSIDERACIONES FINALES: PRINCIPALES APORTACIONES DEL ESTUDIO DE CASO}

Debemos destacar el diagnóstico del caso Maersk como el de un complejo sistema organizacional que mezcla algunos rasgos de cultura fuerte con otros propios de culturas más débiles. Su comportamiento muestra una notable flexibilidad y enormes reflejos como producto de las exigencias del sector marítimo (frenéticas adaptaciones con escasos márgenes de tiempo), que obliga a gran parte de la terminal a trabajar con bastante presión. La compañía danesa combina sus rasgos de cultura planificadora y previsora en las decisiones estratégicas con la capacidad de improvisación exigida por sus clientes, muy conscientes de que los barcos anclados en puerto alguna hora más de lo previsto generan enormes gastos. La necesidad de adaptarse continuamente a los cambios sociales y tecnológicos ha orientado a la compañía positivamente hacia una cultura formativa, si bien los esfuerzos económicos y organizativos por emprender continuas actividades colisionan con la dificultad para gestionar el tiempo de dedicación. Esta labor es aún más difícil que en otras compañías, al contar con el agravio añadido de tener a más de la mitad de la plantilla trabajando por turnos. Asimismo, la empresa ha mostrado algunos problemas en la comunicación vertical que invitan a fomentar la escucha activa, así como un liderazgo de grupos de trabajo más constructivo y sistematizado.

Es importante constatar que la trayectoria de la multinacional Maersk y el Grupo A.P. Möller durante el siglo XX sitúa a su cultura en un auténtico modelo a seguir, entre las consideradas "excelentes". La multinacional danesa es el reflejo de la constancia, entrega, credibilidad, gestión eficaz y espíritu aventurero aplicados a los negocios internacionales. Un modelo a imitar del que otras organizaciones podrían aprender comportamientos. La terminal de Algeciras, en continua expansión y crecimiento, con sus resultados económicos y aportación al empleo local es un buen botón de muestra. 


\section{NOTAS}

${ }^{1}$ Se trata de organizaciones consideradas como referencias a seguir por el resto por su operatividad y resultados, logrados a través de una activa gestión de la cultura de la organización por sus directivos. El calificativo se vincula, en términos generales, a "culturas fuertes". (Deal y Kennedy: 1985)

2 (...) El hombre no puede ser definido solamente por aptitudes innatas, como pretendía la Ilustración, ni solamente por sus modos de conducta efectivos, como tratan de hacer en buena parte las ciencias sociales contemporáneas, sino que ha de definirse por el vínculo entre ambas esferas, por la manera en que la primera se transforma en la segunda. (Geertz: 1973: 24)

${ }^{3}$ Así nos lo hace ver Jeffrey K. Likert en The Toyota way, Madrid, McGraw Hill. 2004; resaltando el peso de la cultura organizacional en la gestión de cambios empresariales, así como la continua importación de técnicas japonesas -sobre todo toyotistas- a la gestión en Estados Unidos.

${ }^{4}$ El desarrollo de las tres vertientes puede verse extensamente en Lucas Marín, A. Sociología de las organizaciones, McGraw Hill. 2002 (pp.211-220).

${ }^{5}$ Este es el caso de Garmendia, J. A. (1994) en sus investigaciones sobre auditorías de cultura corporativa, con un modelo teórico y metodológico que permite retratar la imagen global de una organización, así como comparar diferentes culturas organizativas. Por otra parte, los aplicados por Trinidad Requena, A., Pérez Sánchez, M., (1998) en sus estudios de evaluación han servido también de referencia. A su vez, estos modelos están inspirados en esquemas anteriormente diseñados por autores como Maslow, A (1963), y Parra Luna, F. (1993), aplicados también a evaluación de organizaciones.

${ }^{6}$ La multinacional mantiene la terminología clásica de la Revolución Industrial en la denominación de los grupos de trabajadores: cuello blanco y cuello azul (white collar and blue collar, en las fuentes de la compañía).

${ }^{7}$ Para esta labor se tiene especialmente en cuenta la autocrítica generada por los miembros de la organización y recogida en el trabajo de campo, así como supuestos básicos defendidos por la teoría organizacional y la organización de empresas.

8 Andrews y Basler introducen estos conceptos en 1993. Las culturas fuertes son aquéllas que generan uniformidad en las creencias, valores y actitudes de sus miembros (Ej. el ejército), mientras que las débiles admiten la diversidad entre sus miembros como compatible, al menos, con el logro de las metas de la organización (Ej. la universidad).

${ }^{9}$ Estos códigos (GD3-30. GD3-31...) aparecen en las citas del trabajo de campo cualitativo y responden a una clasificación de la información que facilita el anonimato de los informantes. GD: grupo de discusión; E: entrevista.

${ }^{10}$ En una ocasión un ornitólogo y un biólogo realizaban un complicado trabajo de campo sobre algunas especies de pájaros. Tras numerosas observaciones, a los científicos se le planteaban algunas incógnitas sobre las costumbres de tan interesantes animales. Casualmente, coincidieron con un anciano nativo del lugar, que les aportó un valioso testimonio sobre las costumbres y tendencias de las especies locales, completando las hipótesis iniciales (Eco: 1977).

${ }^{11}$ Barroso Olaya, N. Organización para la flexibilidad en CEPSA. MADOC. Granada, 25 de septiembre 2001(conferencia).

\section{BIBLIOGRAFÍA}

ANDREWS, S.; BASLER, C. y COLLER, X. (2002): "Redes, culturas e identidad en las organizaciones" en REIS, 97: 31-56.

BENNIS, W. (2000): "El fin del liderazgo", en Harvard Deusto Bussines Review, 95: 4-12. 
COLlER, X. y GARVÍA, R. (2004): El análisis de organizaciones, Madrid, Centro de Investigaciones Sociológicas. Monografías, $\mathrm{n}^{\mathrm{o}} 212$ : 59-60.

DEAL, T. y KENNEDY A. (2001a): Culturas corporativas. Ritos y rituales de la vida organizacional, México, FEIL

ECO, U. (1977): Como elaborar una tesis doctoral, Barcelona, Gedisa.

GALPIN, T. (2000): "Conecting culture to organizational change" en HH.RR. Management, 41: 84.

GARMENDIA, J. (1994): Tres culturas: organización y recursos humanos, Madrid, ESIC.

- (2004): "The impact of corporate culture on company performance", Current Sociology, 52: 6-28.

GARVÍA, R.(1997): En el país de los ciegos: La ONCE desde una perspectiva sociológica, Barcelona, Hacer editorial.

GEERTZ, C. (1973): La interpretación de las culturas, Barcelona, Gedisa (primera edición): 23.

GELLES, R. (1994): La corporación japonesa, México, McGraw-Hill.

GIL ESTALLO, M. A. (2004): “Organización vertical versus horizontal”, en ESIC Market, 117: 181197.

GODDAR, A. (1997): Cultura organizacional en fusiones, Tenerife, Universidad de La Laguna.

GÓMEZ CABRANES, L. (1994a): en LUCAS MARÍN, A. (coord.): Sociología para la Empresa (Capítulo 5), pp. 169-185, Madrid, McGraw-Hill.

HOFSTEDE, G. (1999): Culturas y organizaciones, Madrid, Alianza.

HOMBY, O. (1986): With constante care, Copenhague, A.P. Möller Group.

KOTLER, P. (2001): El marketing más eficaz, Deusto, Planeta.

LEAL, A.G. (1991): Conocer la cultura de las organizaciones: una base para la estrategia y el cambio, Madrid, Actualidad editorial.

LIKERT, J.K. (2004): The Toyota way, Madrid, McGraw Hill.

LUCAS MARÍN, A. (2002): Sociología de las organizaciones, Madrid, McGraw-Hill.

OUCHI, W. (1984): La teoría Z ¿Cómo pueden las empresas hacer frente al desafío japonés? Barcelona, Orbis.

PARRA LUNA, F. (1993): El balance integrado de la gestión estratégica: la técnica BIGE, Barcelona, Ediciones Deusto S.A.

PERROW, C. (1996): Sociología de las organizaciones, México, McGraw-Hill.

PETERS, T. J. y WATERMAN, R. H. (1994): En busca de la excelencia, Barcelona, Norma.

RIVERA LATAS, J. y OSENDE, R. (1997): "El hospital del siglo XXI", en Harvard Deusto Business Review, 76: 59-65.

SALA, M. (2000): De la jerarquía a la responsabilidad: el caso Renfe. Barcelona. Ediciones UPC.

SCHEIM, E I. (1995): Organizational culture and leadership, San Francisco, Jossey Bass.

TRINIDAD REQUENA, A. y PÉREZ SÁNCHEZ, M. (1998): "Evaluación de programas de intervención social”, Revista del Ministerio de Trabajo y AA. SS., 10: 113-142.

VALLEJO PEÑA, F.A. (2005): La cultura del trabajo, capítulo 9 en MARTÍNEZ, R. (Coord.) El trabajo en el siglo XXI, pp. 185-207. Granada. Editorial Comares.

- (2007): El análisis de la cultura de una multinacional. El caso Maersk. Granada, Editorial Comares.

WEI CHOO, C. (1999): La organización inteligente, México, Oxford University Press. 


\section{Breve currículo:}

\section{Francisco Alberto Vallejo Peña}

Doctor en Sociología por la Universidad de Granada (2003), ha colaborado en Proyectos de I+D sobre género, inmigración y mercado laboral con el Departamento de Derecho del Estado y Sociología de la Universidad de Málaga. Su experiencia investigadora se ha desarrollado en el campo de la cooperación internacional, con labores en Marruecos (2006-2008) y en el Salvador (1999), en ambos casos desarrollando proyectos para la AECID. Desde el año 2000 centró su interés en el campo de la cultura de empresa, rama en la que ha logrado su mayor difusión como editor con trabajos como El análisis de la cultura de una multinacional (2007), Granada, Ed. Comares; La cultura del trabajo (2005) en Martínez Martín, R. (et al.), El trabajo en el siglo XXI, Granada. Ed. Comares o el artículo "Comunicación horizontal versus vertical: el caso MAERSK" en Revista Internacional de Organizaciones (RIO), AISO, Nº 4, 2008. 